\title{
Persistent gastrointestinal symptoms after correction of malrotation
}

Institute of Child Health, University of London

S P Devane

V V Smith

W M Bisse

B D Lake

P J Milla

Institute of Child Health, University of

Birmingham

$\mathrm{R}$ Coombs

I W Booth

Correspondence to:

Dr S P Devane,

Department of Child Health,

4th Floor, New Ward Block,

King's College Hospital,

London SE5 9RS

Accepted 10 October 1991

S P Devane, R Coombes, V V Smith, W M Bisset, I W Booth, B D Lake, P J Milla

\begin{abstract}
Persistent vomiting, diarrhoea, or intolerance of feeding, are well recognised problems in children after surgical correction of intestinal malrotation. Conversely, intestinal malrotation is a common accompaniment of chronic idiopathic intestinal pseudo-obstruction. We investigated motor activity of the small intestine during fasting in eight children who had persistent vomiting, intolerance of full enteral feeding, or severe diarrhoea after surgical correction of intestinal malrotation. Abnormality of motor function similar to that found in neuropathic pseudo-obstruction was found in seven of the eight patients. Persistence of symptoms after surgical correction of a malrotation is associated with a motility disturbance which seems to be due to a defect of intrinsic enteric innervation. Such a defect may be important in the aetiology of the malrotation.
\end{abstract}

Malrotation of the midgut results from a disturbance of the process by which the herniated midgut rotates and returns to the enlarging fetal abdominal cavity in the twelfth week of gestation. After birth, small intestinal malrotation frequently presents with acute intestinal obstruction and requires surgical intervention. ${ }^{1}$ Symptoms persist after surgery and disordered feeding consistent with abnormal motility is well recognised. As malrotation is common in neuropathic pseudo-obstructive disorders of the midgut, we have studied the motor function of the small intestine in eight children with persistent feeding problems, vomiting, or diarrhoea after surgery in order to investigate whether abnormalities of motility were present which might underlie such problems.

Patients and methods

PATIENTS

Between January 1987 and April 1988, 81 children (aged 1 day to 13 years) with a history of malrotation and intestinal obstruction were seen in the Hospital for Sick Children, London, or the Children's Hospital, Birmingham. Eight (table 1) had persistent severe gastrointestinal symptoms and feeding problems after Ladd's procedure for correction of malrotation, carried out at a median age of 16 months (range 1 month to 13 years). Seven of the eight patients had persistent vomiting. In five of these (patients $1,3,6,7$, and 8 ) vomiting occurred as the enteral intake was increased after surgery, and persisted until the time of referral for investigation. The vomiting was associated with the persistent aspiration of significant amounts of fluid from the stomach, and full enteral feeding could not be established. Three of these five patients were being fed parenterally, one was on continuous gastrostomy feeding, and one was on overnight continuous nasogastric feeding.

In one patient (patient 2), vomiting occurred intermittently and was associated with episodes of abdominal and intestinal distension. One patient (patient 5) had bouts of vomiting associated with severe abdominal pain relieved by cisapride and was intermittently dependent on parenteral nutrition. One patient (patient 4), who had had an ileal resection because of a volvulus, had persistent severe diarrhoea after ingestion of food, not relieved by an exclusion diet, and associated with failure to thrive. One child with vomiting (patient 3 ) had accompanying pyloric stenosis and a congenitally short small intestine. One patient (patient 8) subsequently developed neurofibromatosis. The patients were referred for investigation at a median time of seven months after surgery (range 0.5 months to five years).

The results of the manometric studies were compared with seven control subjects (aged 2.9-13 years, median 6.9 years), undergoing investigation for suspected gut disease, who were found after investigation not to have a gastrointestinal disease. This group consisted of two patients with chest disease undergoing pancreatic function tests, two patients with short stature undergoing jejunal biopsy, one

Table 1 Clinical features of 8 patients with persistent gastrointestinal symptoms after correction of malrotation

\begin{tabular}{lllll}
\hline $\begin{array}{l}\text { Patient } \\
\text { No }\end{array}$ & Symptoms & Route of feeding & $\begin{array}{l}\text { Age at } \\
\text { operation }\end{array}$ & Age at study \\
\hline 1 & Vomiting on feeding & Parenteral and nasogastric & $<1$ week & 6 weeks \\
2 & Intermittent distension and vomiting & Oral & $<1$ week & 9 months \\
3 & Vomiting on feeding & Via gastrostomy & $<1$ week & 5 months \\
4 & Profuse diarrhoea on feeding, failure to thrive & Oral & $<1$ week & 28 months \\
5 & Vomiting on feeding & Parenteral and oral & 8 years & 13 years \\
6 & Vomiting on feeding, constipation & Parenteral & 22 weeks & 7 months \\
7 & Vomiting on feeding & Parenteral & 3 weeks & 5 weeks \\
8 & Vomiting after bolus feeding & Overnight nasogastric & 11 weeks & 23 months \\
\hline
\end{tabular}


patient with episodic abdominal pain undergoing a pancreatic function test, and two patients with a history of episodes of bowel disturbance due to emotional stress in one case and to transient dissaccharide intolerance in the other case.

\section{MANOMETRY}

Motor function of the small intestine after fasting, in particular phase III activity, was used as a probe of the integrity of the enteric nervous system and intestinal smooth muscle. Motor activity was assessed manometrically after a 12 hour fast as previously described. ${ }^{2}$ Activity was recorded for a minimum of three cycles of the migrating motor complex where this was present, or for a minimum period of four hours if not present. Mean migrating motor complex duration (cycle length), mean phase III duration, mean phase III pressure wave frequency, a mean phase III motility index for sum of wave amplitudes/duration), and mean propagation velocity of the phase III activity front were measured at the duodenojejunal flexure.

\section{HISTOLOGY}

In those patients with severe and persistent symptoms where serious neuromuscular disease was suspected, biopsy specimens of the gastrointestinal tract were taken. Rectal suction biopsy specimens were obtained in four subjects (patients 3, 4, 7, and 8) and examined in our laboratory. Full thickness small intestinal biopsy specimens were obtained from four subjects (patients 1, 2, 3, and 5), and were divided into three for fixation in buffered formalin, glutaraldehyde, and for snap freezing. Morphology was assessed on haematoxylin and eosin stained paraffin and cryostat sections, and the amount of connective tissue was estimated using picrosirius and trichrome stains. Acetylcholinesterase activity was detected in snap frozen tissue. Gluteraldehyde fixed tissue was processed for examination by transmission electron microscopy using standard methods.

\section{Results}

\section{MANOMETRY}

The indices measured on the manometric recordings are shown in table 2 , and representa- tive segments of the recordings from three of the patients are shown in the figure. Patient 1 had no detectable motor activity at the time of the study. Patient 2 had very low amplitude pressure waves as shown by a low motility index, with both failure of propagation and retrograde propagation of some phase III activity fronts. Patients 3 and 4 had a slow frequency of contractions in phase III of the migrating motor complex, suggesting abnormal smooth muscle electrical control activity. In addition, patient 3 showed retrograde propagation of the phase III activity front. Patients 5 and 6 showed failure of propagation of some phase III activity fronts. Patient 7 had abnormally slow propagation of the activity front of phase III activity. Patient 8 had normal fasting activity of the small intestine.

\section{HISTOLOGY}

Histological abnormalities in the small intestine were found in three of the four patients in whom full thickness intestinal biopsy specimens were obtained. These abnormalities consisted of distended neuronal axons with ill defined membranes (patient 1), hypoganglionosis of the ileum (patient 2), and vacuolated nerve tracts on electron microscopy (patient 3). Abnormally large nerve trunks in the gastric antrum were found in the fourth of these patients (patient 5). Abnormally large and prominent nerves in the submucosa and myenteric plexus were found in one rectal biopsy (patient 8).

\section{Discussion}

The factors which direct the intestine to rotate when the herniated fetal midgut returns to the abdominal cavity at 12 weeks' gestation are not known. By the time the intestine returns into the fetal abdominal cavity, the smooth muscle cells and the neurones which form the enteric nervous system have migrated into place. Although little is known of functional activity of either the enteric nervous system or intestinal smooth muscle cells at this stage of gestation, there is some knowledge of the development of individual neurotransmitters ${ }^{3}$ and it is known from animal studies that complex maturational processes influenced by the local microenvironment take place before enteric neurones achieve their final mature form. ${ }^{4}$ Malrotation is common in children with pseudo-obstruction, being

Table 2 Indices of motor function of the small intestine obtained from manometric recordings in eight patients with persistent gastrointestinal symptoms after correction of malrotation

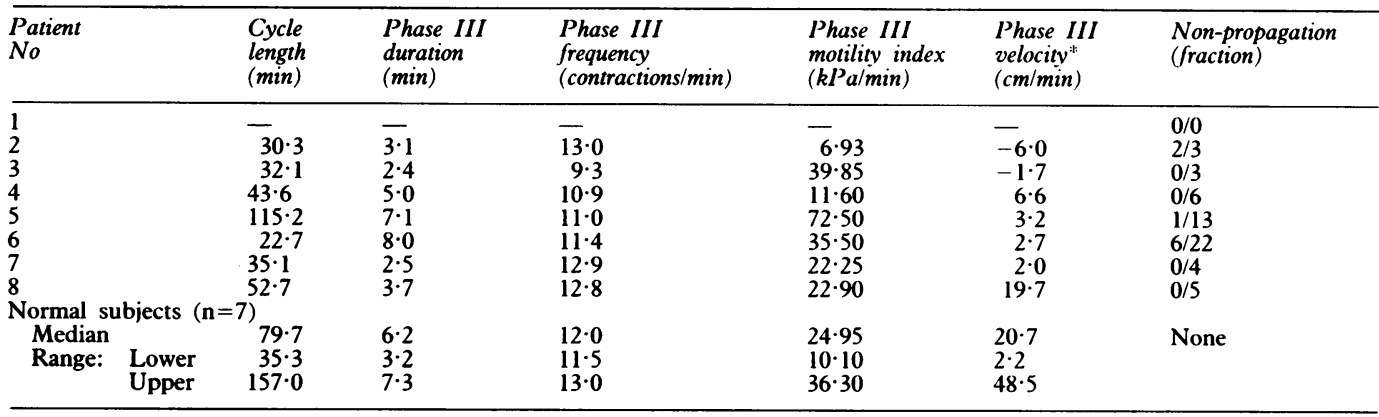

*Negative numbers denote net orad propagation, positive numbers normal aborad propagation. 
A

D1

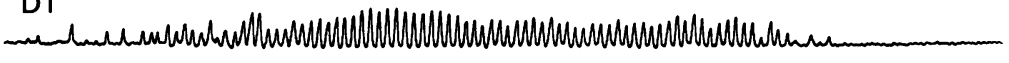

D2

$J$

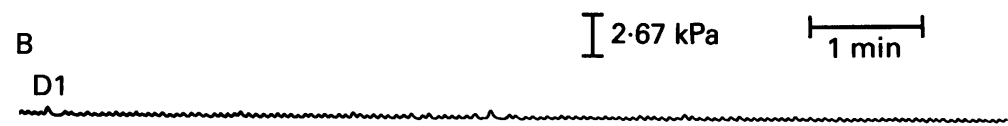

D2

$J$

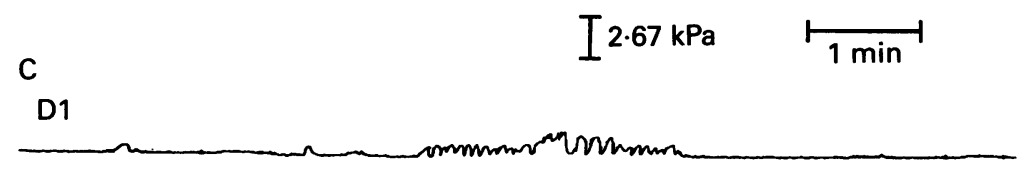

D2

$J$ the migrating motor complex, from $(A)$ patient 8 , showing normal amplitude and frequency of contractions, and normal aborad propagation; $(B)$ patient 4 showing a low frequency of contractions; and $(C)$ patient 2 showing a low amplitude of contractions and failure of propagation of activity to the most distal channel. The ports for channels DI and D2 are positioned sequentially in the duodenum and the port for channel $\mathcal{f}$ is at the duodenojejunal junction; all ports are $5 \mathrm{~cm}$ apart.

associated with approximately $12 \%$ of all types of pseudo-obstructive disorders in which there is disease of the enteric nervous system either from an early age or expressed congenitally. ${ }^{5}$ Thus motor activity may play a part in directing the gut to its correct position within the abdominal cavity. Alternatively, abnormality of the microenvironment in the malrotated intestine may interfere with normal maturation of the neurohumoral control of motor function.

Intestinal motor activity results from the control of inherently contractile smooth muscle cells by the enteric nervous system and polypeptide hormones. The latter are particularly important in the postprandial state whereas fasting activity reflects the activity and integrity of the enteric nervous system. In normal subjects, fasting activity of the small intestine

fluctuates in a cyclical manner over a period of approximately 60 to 90 minutes. This cycle is called the migrating motor complex and it consists of three phases, phases I, II, and III. Phase III is a period of rhythmic contractile activity at a frequency of 11-12 cycles per minute (figure). It is followed by a period of quiescence, phase I, and preceeded by irregular contractions, phase II. This motor complex migrates aborally along the small intestine.

The manometric abnormalities present in patients with pseudo-obstruction have been described in both adults ${ }^{6}$ and children. ${ }^{7-9}$ In the former study, the most common abnormality found was abnormal propagation and/or conformation of the migrating motor complex (found in 25 of the 42 subjects) together with bizarre waveforms. In the latter studies, in children, the fasting abnormalities included in addition absent migrating motor complexes and very low amplitude or absent contractions. In those manometric investigations where full thickness intestinal biopsies have also been done and adequately studied, very low amplitude contractions with poorly differentiated phase III periods have been associated with smooth muscle disease. ${ }^{8}$ In contrast, contractile activity of normal or increased amplitude where there is disorganisation, especially loss of cyclic activity, lack of propagation, abnormal propagation or presence of bizarre clusters of contractions, is usually found in patients with neuropathic disorders. ${ }^{9}$

Five of the eight patients in our series had very abnormal motor activity. Four had abnormal propagation and/or conformation of phase III of the migrating motor complex (with or without a low amplitude of contraction), similar to the abnormalities characterised by Stanghellini et $a l^{6}$ and by Wozniak et al. ${ }^{9}$ One had no motor activity, which was similar to one of the patterns described by Hyman et al. ${ }^{7}$ Of the other three patients, one had abnormally slow propagation of the phase III complex, and one had an abnormally slow frequency of contraction in phase III, when compared with the values obtained in subjects without gastrointestinal disease. These findings suggest that the majority of our patients who continued to have gastrointestinal problems after correction of malrotation have disturbances of motor function similar to those found in patients with idiopathic intestinal pseudo-obstruction, particularly those with neuropathic disease involving the enteric nervous system.

The histopathology of neuromuscular disease of the intestine has been the subject of a number of reports ${ }^{1011}$ and both muscle and nerve disorders have been described. A recent report of visceral neuropathies, excluding aganglionosis of the colon and Hirschsprung's disease, summarised the most common intestinal conditions. $^{12}$ Such conditions included hypoganglionosis, hyperganglionosis or neuronal intestinal dysplasia, glial cell hyperplasia, and absent or diminished argyrophil neurones associated with pyloric stenosis, a short small intestine, and malrotation. ${ }^{13}$

In the present study, one of our patients had hypoganglionosis of the small intestine whose 
proximal extent was not defined, and had total colonic aganglionosis. Two of our patients had abnormal enteric nervous on electron microscopy. Therefore three of the four patients who had full thickness biopsies had abnormalities in the small intestine previously associated with pseudo-obstruction. The significance of the gastric antral abnormality in the fourth patient (patient 5) is uncertain.

Intestinal neuronal dysplasia can be diagnosed from suction biopsies of the rectum by assessment of acetylcholinesterase-positive nerve cells in the lamina propria, hyperplasia of submucosal neurones and the presence of displaced neurones within the lamina propria. ${ }^{14}$ In our series of patients, rectal biopsies were helpful in excluding intestinal neuronal dysplasia and in demonstrating the abnormality in patient 8 . It may be that the large nerve trunks seen in this patient, who later developed neurofibromatosis, may indicate early evidence of the spectrum of disorders which include glial cell hyperplasia ${ }^{12}$ and intestinal neuronal dysplasia.

One child (patient 4) had a short small intestine due to surgical resection and presented with diarrhoea. There are no published manometric studies of motor function of the small intestine in children with short gut syndrome. Therefore it is not possible to know whether the abnormality found in this case (a slow frequency of contraction in phase III) is secondary to the resection.

Abnormalities of motor function may occur in the period immediately after intestinal surgery. Prolonged ileus is not uncommon in the period immediately after surgery for malrotation. In six of our eight cases the manometric study was performed more than 10 weeks after surgery. The long term effect of surgery on small intestinal activity has not been systematically investigated in children, but in adults who had a proctocolectomy and ileoanal anastomosis, jejunoileal fasting activity was not greatly altered 4-24 months after the operation. ${ }^{15}$ We suggest that, in view of the nature of Ladd's procedure and of these adult studies, it is unlikely that the motor abnormalities were caused by surgery in these patients.

Two of the subjects (patients 6 and 7) have now died having failed to take enteral feeding without resultant vomiting, two subjects (patients 1 and 2) are well on parenteral feeding, one (patient 5) is well on enteral feeding and cisapride, and the three remaining subjects suffer intermittent gastrointestinal symptoms on enteral feeding.

Our findings support the notion that in those children where symptoms of gastrointestinal disturbance continue despite surgical correction there is disordered intestinal motor activity. The pattern of disordered activity suggests that it results from either disorder or disease of the enteric nervous system. We therefore postulate that in some children with malrotation aberrant early development of either the enteric nervous system or its microenvironment results both in malrotation of the intestine and its later disordered function.

This project was supported, in part, by the Janssen Research Foundation, and this paper was presented in part to the European Society for Paediatric Gastroenterology and Nutrition, Copenhagen, May 1988.

1 Rees JR, Redo SF. Anomalies of intestinal rotation and fixation. Am $\mathcal{F}$ Surg 1968;116:834-4

2 Fenton T, Harries JT, Milla PJ. Disordered small intestinal motility: a rational basis for toddler's diarrhoea. Gut 1983;24:897-903.

3 Chavyvialli JA, Paulin C, Descos F, Dubois PM. Ontogeny of vasoactive intestinal peptide in the human fetal digestive tract. Regul Pept 1983;5:245-56.

4 Le Douarin NM. The ontogeny of the neural crest in avian embryo chimeras. Nature 1980;286:663-9.

5 Vargas JH, Sachs P, Ament ME. Chronic ideopathic pseudoobstruction in pediatrics. I Pediatr Gastroenterol Nutr obstruction in

6 Stanghellini V, Camilleri M, Malagelada J-R. Chronic idiopathic intestinal pseudo-obstruction: clinical and intestinal manometric findings. Gut 1987;28:5-12.

7 Hyman PE, McDiarmid SU, Napolitano J, Abrams CE, Tomomasa T. Antroduodenal manometry in children with chronic intestinal pseudo-obstruction. $\mathcal{F}$ Pediatr 1988;112: 899-905.

8 Milla PJ, Lake BD, Spitz L, Nixon HH, Harries JT, Fenton TR. Chronic idiopathic intestinal pseudo-obstruction in infancy: a smooth muscle disease. In: Labb G, Bortolotti M, eds. Gastrointestinal motility. Verona: Cortina International, 1983:125-31.

9 Wozniak E, Fenton TR, Milla PJ. Fasting small intestinal motor activity in chronic idiopathic intestinal pseudoobstruction. Pediatr Res 1984;18:1060.

10 Rohrmann CA, Ricci MT, Krishnamurthy S, Schuffler MD. Radiologic and histologic differentiation of neuromuscular disorders of the gastrointestinal tract: visceral myopathies, visceral neuropathies, and progressive systemic sclerosis. AfR 1984;143:933-41.

11 Krishnamurthy S, Schuffler MD. Pathology of neuromuscular disorders of the small intestine and colon. Gastroenterology 1987;93:601-39.

12 Navarro J, Sonsino E, Boige N, et al. Visceral neuropathies responsible for chronic intestinal pseudo-obstruction syndrome in pediatric practice: analysis of 26 cases. $\mathcal{f}$ Pediatr Gastroenterol Nutr 1990;11:179-95.

13 Tanner MS, Smith B, Lloyd JK. Functional intestinal obstruction due to deficiency of argyrophil neurons in the myenteric plexus: familial syndrome presenting with short small bowel, malrotation, and pyloric hypertrophy. Arch Dis Child 1976;51:837-41

14 Puri P, Lake BD, Nixon HH, Mishalany H, Claireaux AE. Neuronal colonic dysplasia: an unusual association of Hirschsprung's disease. $\mathcal{F}$ Pediatr Surg 1977;12:681-5.

15 Stryker SJ, Borody T], Phillips SF, Kelly KA, Dozois RR, Beart RW Jr. Motility of the small intestine after proctocolectomy and ileal pouch anastomosis. Ann Surg 1985;201: 351-6. 